Pathologe 2010 · [Suppl 2] 31:134-137 DOI 10.1007/s00292-010-1339-5

Online publiziert: 18. August 2010

(c) Springer-Verlag 2010

\author{
F. Haller ${ }^{1,4}$. D.J. Zhang ${ }^{2} \cdot$ C. Löbke ${ }^{2} \cdot$ A. von Heydebreck ${ }^{3} \cdot$ U. Korf ${ }^{2} \cdot$ L. Füzesi ${ }^{4}$. \\ Ö. Sahin ${ }^{2}$ \\ ${ }^{1}$ Institut für allgemeine Pathologie und pathologische Anatomie, \\ Universitätsklinikum Freiburg \\ ${ }^{2}$ Deutsches Krebsforschungszentrum, Heidelberg \\ ${ }^{3}$ Merck KGaA, Darmstadt \\ ${ }^{4}$ Institut für Pathologie, Universitätsmedizin Göttingen
}

\title{
Multilayer-Analyse der Signaltransduktion und Zellzykluskontrolle in GIST
}

\section{Identifizierung neuer Interaktionspartner mit differenzieller Regulation}

Gastrointestinale Stromatumoren (GIST) treten im gesamten Gastrointestinaltrakt auf, wobei GIST des Magens ein generell weniger aggressives biologisches Verhalten zeigen als GIST des Dünn- und Dickdarms [1]. Histomorphologisch können bei Magen-GIST und Dünndarm-GIST verschiedene Wachstumsmuster unterschieden werden. Auf molekulargenetischer Ebene können GIST nach dem Genotyp in GIST mit einer KIT-Mutation, GIST mit einer PDGFRA-Mutation

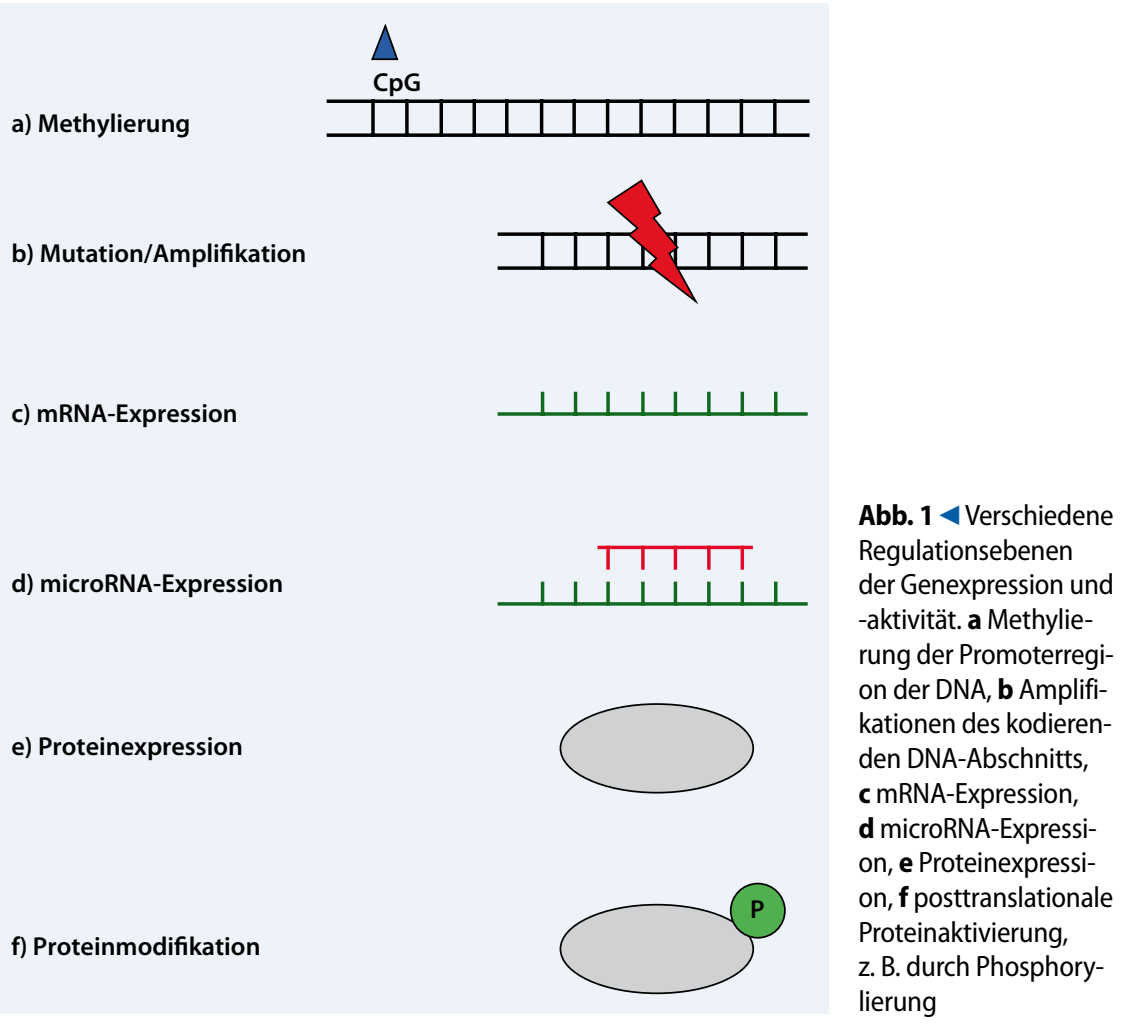

sowie in so genannte Wildtyp-GIST unterschieden werden [2]. Genexpressionsstudien auf mRNA-Ebene konnten zeigen, dass sowohl die anatomische Lokalisation als auch der Genotyp bei GIST mit einer differenziellen Genexpression assoziiert sind.

Die Regulation der Aktivität eines Genprodukts ist komplex und findet auf verschiedenen Ebenen statt ( $\bullet$ Abb. 1 a-f). Die Methylierung der Promoterregion reguliert die Expression auf mRNA-Ebene (• Abb. 1 a). Amplifikationen führen zu einer gesteigerten Transkription (- Abb. 1 b). Je mehr mRNA transkribiert wird (• Abb. 1 c), desto mehr Protein wird erzeugt (- Abb. 1 e). MicroRNAs sind an dieser Stelle eingreifende, zwischengeschaltete Feinregulatoren, welche die mRNA wieder abbauen können, bevor das Protein gebildet werden kann (- Abb. 1 d). Die Aktivität vieler Proteine wird häufig zusätzlich posttranslational reguliert, Z. B. über Modifikationen wie Phosphorylierungen (• Abb. 1 f).

Ziel dieser Studie war es, Daten auf verschiedenen Ebenen der Genregulation bei GIST zu erheben und unter Berücksichtigung der bei GIST bekannten Signalwege zusammenzuführen, um neue und funktionell relevante Geninteraktionen identifizieren zu können. 


\section{Analyse der Promotermethylierung}

Die Methylierung der Promoterregionen von 807 krebsrelevanten Genen (insgesamt 1505 CpG-Loci) wurde in 61 primären GIST mittels der „Illumina Golden Gate Plattform“ („Illumina Golden Gate Cancer Methylation Panel") analysiert [3]. Die Methylierung der einzelnen CpG-Loci wurde mit den klinisch-pathologischen Parametern und dem Follow-up korreliert. Bei GIST mit einer KIT-Mutation waren im Vergleich zwischen MagenGIST und Dünndarm-GIST 115 CpG-Loci differenziell methyliert. Im Gegensatz dazu konnten im Vergleich von GIST des Magens mit einer KIT- bzw. PDGF$R A$-Mutation 102 differenziell methylierte CpG-Loci identifiziert werden, die signifikant mit dem Genotyp assoziiert waren. 235 CpG-Loci waren im Vergleich von GIST mit < 5 vs. $>5$ Mitosen pro 50 hochauflösende Gesichtsfelder (HPFs) unterschiedlich methyliert.

\section{Analyse der mRNA-Expression}

Die mRNA-Expression wurde zunächst mittels „Whole-Genome Oligonucleotid Arrays" („Illumina Golden Gate") in 24 GIST untersucht [3]. In der Analyse waren 144 Gene im Vergleich von Magen-GIST mit KIT- bzw. PDGFRA-Mutation differenziell exprimiert, während die Expression von 531 Genen mit der anatomischen Lokalisation bzw. von 392 Genen mit der Mitosenanzahl assoziiert war. Die differenzielle Expression wurde exemplarisch für 10 Gene mittels quantitativer „Real-time-RT-Polymerase-Ketten-Reaktion (PCR) “ in 61 GIST bestätigt $[3,4]$.

\section{Analyse der microRNA-Expression}

Die microRNA-Expression wurde zunächst in 12 GIST mittels eines Oligonukleotid-Arrays (miRXplore Microarray, Miltenyi Biotec $\mathrm{GmbH}$ ) analysiert, wobei der Array 734 humane microRNAs abdeckte [5]. Die Expression von 16 microRNAs war mit der anatomischen Lokalisation assoziiert, während 21 microRNAs eine differenzielle Expression in Zusammenhang mit dem Genotyp aufwiesen. Die differenzielle Expression von 4 microRNAs

Pathologe 2010 · [Suppl 2] 31:134-137 DOI 10.1007/s00292-010-1339-5

c) Springer-Verlag 2010

F. Haller · D.J. Zhang · C. Löbke · A. von Heydebreck · U. Korf · L. Füzesi · Ö. Sahin

Multilayer-Analyse der Signaltransduktion und

Zellzykluskontrolle in GIST. Identifizierung neuer

Interaktionspartner mit differenzieller Regulation

\section{Zusammenfassung}

Um neue Interaktionspartner und diagnostisch, prognostisch und therapeutisch relevante Unterschiede in der Regulation der Genexpression bei gastrointestinalen Stromatumoren (GIST) zu identifizieren, wurden in identischen Patientenproben parallel die Methylierung, die mRNA-Expression, die microRNA-Expression, die Proteinexpression und die Proteinphosphorylierung analysiert. Die Daten wurden in einer Multilayer-Analyse zusammengeführt und sowohl untereinander als auch mit klinisch-pathologischen Parametern korreliert. Differenziell regulierte Gene wurden mit bereits bekannten Signalwegen bei GIST verglichen und in einer funktionell orientierten Übersicht zusammengeführt, was neue Erkenntnisse über die Genregulation bei GIST im Zusammenhang ermöglichte.

Schlüsselwörter

Gastrointestinale Stromatumoren .

Methylierung $\cdot$ mRNA $\cdot$ microRNA $\cdot$ Protein

\section{Multilayer analysis of signal transduction and cell cycle control in GIST. Indentifying new interaction partners with differential regulation}

\section{Abstract}

To identify new interactions as well as diagnostically, prognostically and therapeutically relevant differences in the regulation of gene expression in gastrointestinal stromal tumors (GISTs), we analyzed the methylation status, mRNA expression, microRNA expression, protein expression and protein phosphorylation in parallel in identical tumor tissue samples. The data were analyzed in a multilayer approach and were correlated to each other and to clinico-pathological pa- rameters. Differentially regulated genes were mapped to signal transduction pathways which are already known to play a major role in GISTs. A functionally orientated overview of the different data layers was constructed, which enabled new insights into gene regulation in GISTs.

\section{Keywords}

Gastrointestinal stromal tumors · Methylation · RNA, messenger · microRNA · Protein 


\section{Referate Preisträger: Posterpreisträger 2010}

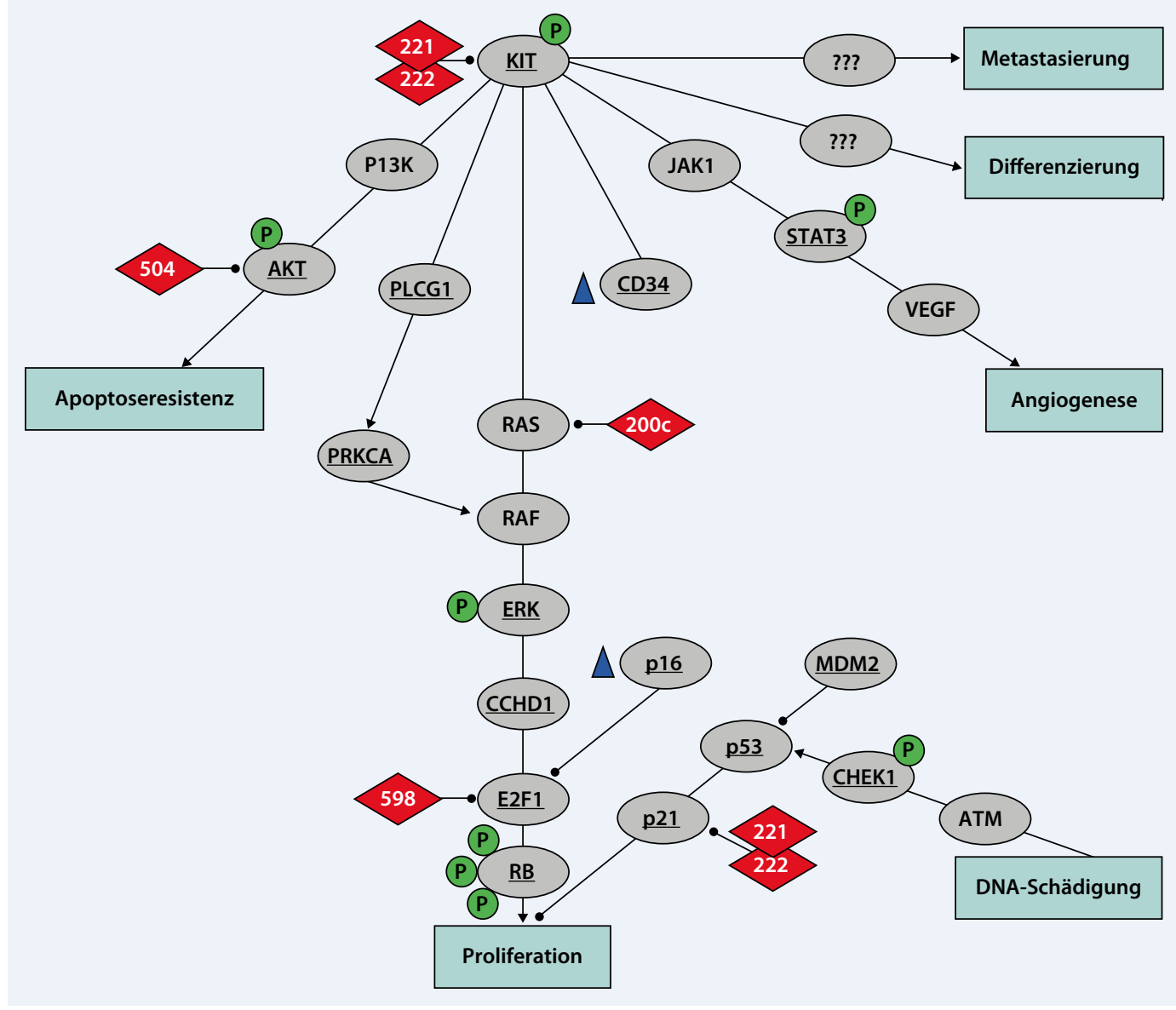

Abb. $2<$ Übersicht über differenzielle Regulation von bekannten Signalwegen bei GIST. Proteine sind als Kreise dargestellt, microRNAs als Rauten. Unterstrichene Proteine zeigten eine gute Korrelation zwischen mRNA- und Proteinexpression sowie eine differenzielle Expression in Assoziation mit verschiedenen klinisch-pathologischen Parametern. $P$ zeigt Phosphorylierungsstellen an. Das blaue Dreieck zeigt Gene an, deren mRNA-Expression stark mit der Promotermethylierung korrelierte

wurde mittels quantitativer Real-time-RTPCR in 61 GIST bestätigt.

\section{Analyse der Proteinexpression}

Die Proteinexpression wurde auf 2 unterschiedliche Arten untersucht: Von 61 GIST wurde Zellextrakt isoliert und auf Objektträger gespottet, und die Proteinmenge von 50 verschiedenen Proteinen bzw. Phosphoproteinen wurde quantitativ mittels so genannter „Reverse-PhaseProtein-Arrays" bestimmt $[6,7]$. Parallel wurden von 198 GIST aus paraffineingebettetem Tumorgewebe „Tissue-Microarrays" hergestellt und ebenfalls 50 Proteine mittels Immunhistochemie untersucht $[8$, 9]. Um eine exakte Analyse der immunhistochemischen Färbungen zu ermöglichen, wurden von jedem Tumor 6 Stanzen gefärbt, digital fotografiert und mittels einer selbstentwickelten Software quantitativ ausgewertet [10].
Multilayer-Analyse

Die bei GIST bereits nachgewiesenen aktivierten Signalwege wurden aus Publikationen herausgesucht. Die verschiedenen eigenen Datensätze wurden zusammengeführt und auf potenzielle Geninteraktionen auf den verschiedenen Regulationsebenen untersucht. Informationen über bereits bekannte Interaktionen wurden aus verschiedenen Datenbanken (KEGG, miRBase, TargetScan) extrahiert und in die Analyse aufgenommen. Es zeigte sich eine teilweise hochsignifikante differenzielle Expression bzw. Aktivierung zentraler Signalwege in Assoziation mit klinisch-pathologischen Parametern wie der anatomischen Lokalisation, dem Genotyp oder der Mitosenanzahl (• Abb. 2). Insgesamt konnten sowohl die Bedeutung bereits bekannter Signalwege bei GIST bestätigt werden als auch neue, potenziell diagnostisch, prognostisch oder therapeutisch relevante Interaktionen auf verschiedenen Ebenen identifiziert werden.

\section{Fazit für die Praxis}

Bei GIST ist die Aktivierung zentraler Signalwege teilweise mit der anatomischen Lokalisation, dem Genotyp und der Mitosenanzahl korreliert, wobei auf verschiedenen Ebenen der Genregulation Unterschiede bestehen. Eine umfassende Analyse der Genexpression bzw. Proteinaktivität auf verschiedenen Ebenen an den gleichen Patientenproben hat den Vorteil, ein umfassenderes, funktionell relevantes Bild der Regulation zu liefern, und kann neue Interaktionen aufdecken. Diese Multilayer-Methode wird helfen, in der Zukunft weitere diagnostische, prognostische und therapeutisch relevante Faktoren bei GIST zu identifizieren.

\section{Korrespondenzadresse \\ PD Dr. F. Haller}

Institut für allgemeine Pathologie und pathologische Anatomie, Universitätsklinikum Freiburg Breisacher Str. 115a, 79106 Freiburg florian.haller@uniklinik-freiburg.de 
Interessenkonflikt. Der korrespondierende Autor weist auf folgende Beziehungen hin: Reisekostenübernahme und Referententätigkeit für Novartis Oncology mit Honorar; Forschungsdrittmittel von Novartis Oncology.

\section{Literatur}

1. Miettinen M, Lasota J (2006) Gastrointestinal stromal tumors: review on morphology, molecular pathology, prognosis and differential diagnosis. Arch Pathol Lab Med 130:1466-1478

2. Lasota J, Miettinen M (2008) Clinical significance of oncogenic KIT and PDGFRA mutations in gastrointestinal stromal tumours. Histopathology 53:245266

3. Haller $\mathrm{F}$ et al (o J) manuscript in preparation

4. Haller F, Gunawan B, Heydebreck A von et al (2005) Prognostic role of E2F1 and members of the CDKN2A network in gastrointestinal stromal tumors. Clin Cancer Res 11:6589-6597

5. Haller F, Heydebreck A von, Zhang JD et al (2010) Localization- and mutation-dependent microRNA (miRNA) expression signatures in gastrointestinal stromal tumours (GISTs), with a cluster of coexpressed miRNAs located at 14q32.31. J Pathol 220:71-86

6. Haller F, Löbke C, Ruschhaupt M et al (2008) Increased KIT signalling with upregulation of cyclin D correlates to accelerated proliferation and shorter disease-free survival in gastrointestinal stromal tumours (GISTs) with KIT exon 11 deletions. J Pathol 216:225-235

7. Haller F, Löbke C, Ruschhaupt M et al (2008) Loss of $9 p$ leads to p16INK4A downregulation and enables RB/E2F1-dependent cell cycle promotion in gastrointestinal stromal tumours (GISTs). J Pathol 215:253-262

8. Haller F, Agaimy A, Cameron S et al (2010) Expression of p16INK4A in gastrointestinal stromal tumours (GISTs): two different forms exist that independently correlate with poor prognosis. Histopathology 56:305-318

9. Haller F, Cortis J, Helfrich J et al (o J) Epithelioid/ mixed phenotype in gastrointestinal stromal tumors (GISTs) with KIT mutation from the stomach is associated with accelerated passage of late phases of the cell cycle and shorter disease-free survival. Mod Pathol (manuscript accepted)

10. Cameron S, Haller F, Dudas J et al (2008) Immune cells in primary gastrointestinal stromal tumors. Eur J Gastroenterol Hepatol 20:327-334

\section{Posterpreise 2010}

\section{Preis}

S.K. Scheel ${ }^{1}$, B. Das ${ }^{2}$, T. Kirchner ${ }^{1}$, A. Jung ${ }^{1}$

${ }^{1}$ Pathologisches Institut, LMU München

${ }^{2}$ Division of Oncology, Stanford School of Medicine, USA

Expression von FGF-2 ist essentiell für die Aufrechterhaltung von Tumorstammzell-Eigenschaften in humanen kolorektalen Tumor Zelllinien

\section{Preis}

L.M. Pawella ${ }^{1}$, M. Hashani ${ }^{1}$, R. Zimbelmann², H. Heid², P. Schirmacher ${ }^{1}$, B.K. Straub ${ }^{1}$

${ }^{1}$ Pathologisches Institut, Universitätsklinik Heidelberg

${ }^{2}$ Helmholtz-Gruppe Zellbiologie, DKFZ Heidelberg

Effekte einer Induktion und siRNA-mediierten Herunterregulation Lipidtropfenassoziierter Proteine in hepatozytären Zellkulturmodellen

E.C. Obermann, N. Müller, A. Rufle, T. Menter, S. Dirnhofer, A. Tzankov Institut für Pathologie, Universität Basel, Schweiz

Klonale Verwandtschaft rezidivierender Hodgkin Lymphome

\section{Preis}

M. Rezaei ${ }^{2}$, M. Labelle ${ }^{1}$, A. Kettelhake ${ }^{1}$, A. Kuzmanov ${ }^{1}$, G. Baretton' ${ }^{1}$, G. Breier ${ }^{1}$

${ }^{1}$ Department of Pathology, University of Technology, Dresden, Germany

${ }^{2}$ MIT Center for Cancer Research, Cambridge, USA

Die Tumorprogression im Mammakarzinom ist assoziiert mit der differentiellen Expression von VE-Cadherin und HIF Hydroxylasen

F. Haller ${ }^{1}$, D. Zhang ${ }^{2}$, A. von Heydebreck ${ }^{3}$, L. Füzesi' ${ }^{4}$, O. Sahin ${ }^{2}$

${ }^{1}$ Institut für Pathologie, Universitätsklinikum Freiburg

${ }^{2}$ German Cancer Research Center, Heidelberg

${ }^{3}$ Merck KgAA, Darmstadt

${ }^{4}$ Institut für Pathologie, Universitätsklinik Göttingen

Multilayer Analyse der Signaltransduktion und Zellzykluskontrolle auf mRNA-, miRNA- und Proteinexpressionsebene zeigt prognostisch relevante Interaktionen in GIST

Das Preisgeld für die Posterpreise wurde freundlicherweise vom Bundesverband Deutscher Pathologen e.V., Berlin, zur Verfügung gestellt. 